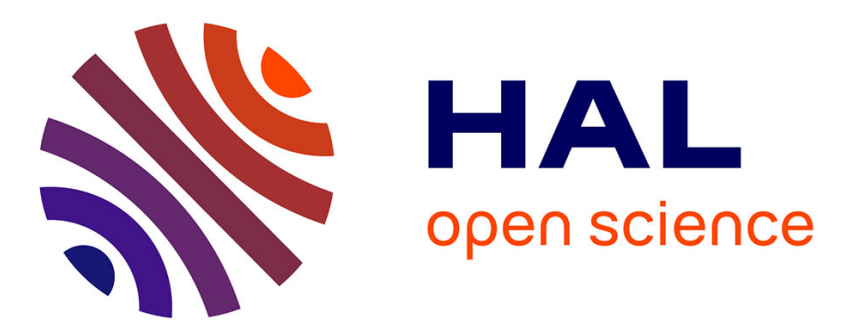

\title{
X-ray absorption near edge spectroscopy study of warm dense $\mathrm{MgO}$
}

\author{
R. Bolis, J.-A. Hernandez, V. Recoules, M. Guarguaglini, F. Dorchies, N. \\ Jourdain, A. Ravasio, T. Vinci, E. Brambrink, N. Ozaki, et al.
}

\section{- To cite this version:}

R. Bolis, J.-A. Hernandez, V. Recoules, M. Guarguaglini, F. Dorchies, et al.. X-ray absorption near edge spectroscopy study of warm dense MgO. Physics of Plasmas, 2019, 26 (11), pp.112703. 10.1063/1.5105390 . hal-03009014

\section{HAL Id: hal-03009014 https://hal.science/hal-03009014}

Submitted on 17 Nov 2020

HAL is a multi-disciplinary open access archive for the deposit and dissemination of scientific research documents, whether they are published or not. The documents may come from teaching and research institutions in France or abroad, or from public or private research centers.
L'archive ouverte pluridisciplinaire HAL, est destinée au dépôt et à la diffusion de documents scientifiques de niveau recherche, publiés ou non, émanant des établissements d'enseignement et de recherche français ou étrangers, des laboratoires publics ou privés. 


\title{
X-ray absorption near edge spectroscopy study of warm dense MgO
}

\author{
Cite as: Phys. Plasmas 26, 112703 (2019); doi: 10.1063/1.5105390 \\ Submitted: 27 April 2019 - Accepted: 3 October 2019 . \\ Published Online: 7 November 2019
}

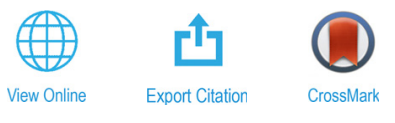

\begin{abstract}
R. Bolis, ${ }^{1,2, a)}$ (D J.-A. Hernandez, ${ }^{1,2}$ (D) V. Recoules, ${ }^{3}$ M. Guarguaglini, ${ }^{1,2}$ (iD F. Dorchies, ${ }^{4}$ (D) N. Jourdain, ${ }^{4}$ A. Ravasio, ${ }^{1,2}$ T. Vinci, ${ }^{1,2}$ (D) E. Brambrink, ${ }^{1,2}$ N. Ozaki, ${ }^{5,6}$ J. Bouchet, $^{3}$ F. Remus, ${ }^{3}$ R. Musella, ${ }^{7}$ S. Mazevet, ${ }^{7}$ N. J. Hartley, ${ }^{5,8}$ (iD F. Guyot, ${ }^{9}$ and A. Benuzzi-Mounaix ${ }^{2,10}$
\end{abstract}

\author{
AFFILIATIONS \\ 'LULI - CNRS, Ecole Polytechnique, CEA, Université Paris-Saclay, Palaiseau cedex 91120, France \\ ${ }^{2}$ Sorbonne Universités, CNRS, Laboratoire d'utilisation des Lasers Intenses (LULI), Campus Pierre et Marie Curie, place Jussieu, \\ Paris cedex 75005 , France \\ ${ }^{3}$ CEA, DAM, DIF, Arpajon 91290, France \\ ${ }^{4}$ Université de Bordeaux, CNRS, CEA, CELIA (Centre Lasers Intenses et Applications), UMR 5107, Talence 33400, France \\ ${ }^{5}$ Graduate School of Engineering, Osaka University, Suita 565-0871, Japan \\ ${ }^{6}$ Photon Pioneers Center, Osaka University, Suita 565-0871, Japan \\ ${ }^{7}$ LUTH, Observatoire de Paris, CNRS, Université Paris Diderot, Meudon 92195, France \\ ${ }^{8}$ Helmholtz-Zentrum Dresden-Rossendorf, Dresden 01328, Germany \\ ${ }^{9}$ Institut de Minéralogie, de Physique des Matériaux et de Cosmochimie, UMR CNRS 7590, Sorbonne Universités-Université Pierre et \\ Marie Curie, CNRS, Muséum National d'Histoire Naturelle, IRD, Paris 75005, France \\ ${ }^{10}$ LULI - CNRS, Ecole Polytechnique, CEA, Université Paris-Saclay, F-91128 Palaiseau cedex 91120, France \\ a) Author to whom correspondence should be addressed: riccardomaria.bolis@gmail.com
}

\begin{abstract}
We report time-resolved X-ray Absorption Near Edge Spectroscopy (XANES) measurements of warm dense MgO. We used a high power nanosecond pulse to drive a strong uniform shock wave into an MgO sample, and a picosecond pulse to generate a broadband X-ray source near the $\mathrm{Mg} \mathrm{K}$-edge. We used this setup to obtain XANES spectra across a large area of the phase diagram, with densities up to $6.8 \mathrm{~g} / \mathrm{cc}$ and temperatures up to $30000 \mathrm{~K}$, conditions at which no prior investigations of electronic and ionic structure exist. Our XANES results, together with quantum molecular dynamic simulations, demonstrate that the sample metallizes due to the bandgap closure as it melts, after which it shows typical behavior for a disordered ionic liquid.
\end{abstract}

Published under license by AIP Publishing. https://doi.org/10.1063/1.5105390

\section{INTRODUCTION}

Understanding the physical properties of matter at extreme conditions of pressure, density, and temperature is relevant across many different fields of physics, including materials science, inertial confinement fusion, and planetology. The behavior of $\mathrm{MgO}$ under such conditions is particularly important, due its unique properties-most notably, that it shows no phase transitions up to pressures of $500 \mathrm{GPa}^{1}$ For this reason, it is often used as a pressure medium in high pressure solid media devices. It is also an important material in geophysics, ${ }^{2}$ medical materials ${ }^{3}$ and, due to its high melting temperature, in industrial applications, such as for making catalysts, refractory materials, and optically transparent glass ceramics.
Many of these applications would benefit from a precise understanding of the structure and behavior of liquid $\mathrm{MgO}$ under conditions of high pressure and temperature-the warm dense matter (WDM) regime. In industrial applications, for example, it is essential in order to optimize the processes of making new materials, ${ }^{18}$ and in geophysics better characterizing the melting allows better constraints of the solidus in the Earth's lower mantle. Beyond our planet, magnesiumiron oxides and magnesium-iron rich silicates such as $(\mathrm{Fe}, \mathrm{Mg}) \mathrm{SiO}_{3}$ and $\left.(\mathrm{Fe}, \mathrm{Mg})_{2} \mathrm{SiO}_{4}\right)$ and their pure end members- $\mathrm{SiO}_{2}, \mathrm{MgO}$, and $\mathrm{FeO}$-are also believed to be major constituents of mantles in terrestrial extrasolar planets. Understanding their equations of state, phase diagrams, and structural and electronic properties is therefore essential 
for modeling the internal structures, dynamics, and evolution of terrestrial planets. ${ }^{5}$ Liquid silicates may even be able to drive dynamos, ${ }^{6}$ either in the early history of the Earth, ${ }^{7}$ or in extrasolar planets with larger and longer-lived magma oceans. ${ }^{8}$ However, the significance of these effects is still poorly understood precisely because of the lack of knowledge of electronic properties at the extreme conditions found in magma oceans.

X-ray absorption near-edge spectroscopy (XANES) allows changes in the electronic structure of materials to be observed directly. Previous results from $\mathrm{SiO}_{2}$ demonstrated that the electronic bandgap closes into a pseudogap with an increasing temperature, leading to a semimetallic behavior as the $\mathrm{Si}$ and $\mathrm{O}$ atoms lose correlation. ${ }^{9,10}$ The results were compared with ab initio calculations, ${ }^{11-13}$ showing a good agreement and demonstrating the reliability of $a b$ initio calculations to describe metallization. Liquid $\mathrm{SiO}_{2}$ has also been shown to undergo structural changes with increasing pressure, with an increase in the coordination number from 4 to 9 from 2 to $8 \mathrm{~g} / \mathrm{cm}^{3}$. 10

The electronic and structural properties of liquid $\mathrm{MgO},(\mathrm{Mg}$, $\mathrm{Fe}) \mathrm{O}$, and $\mathrm{MgSiO}_{3}$ have been the subject of extensive theoretical study, ${ }^{13-19}$ but until now, no direct experimental data has been reported at high pressures. Previous shock compression experiments probed the electronic properties of $\mathrm{MgO}, \mathrm{MgSiO}_{3}$, and $\mathrm{Mg}_{2} \mathrm{SiO}_{4}$ only indirectly, by measuring reflectivity along the principal Hugoniot and calculating the electronic conductivity using Drude models, ${ }^{20-23}$ while the XANES data is restricted to the ambient density, at temperatures up to a few thousand degrees. ${ }^{24,25}$ In this article, we report timeresolved XANES spectroscopy measurements of warm dense $\mathrm{MgO}$ up to $30000 \mathrm{~K}$ and $6.5 \mathrm{~g} / \mathrm{cm}^{3}$. The data is supported by $A b$ Initio Molecular Dynamics (AIMD) simulations, with the bandgap closure and subsequent liquid behavior observed in the XANES spectra.

\section{EXPERIMENTAL DETAILS}

In order to obtain XANES spectra at WDM conditions, we used an experimental setup similar to that in the recent studies on $\mathrm{Al}$ and $\mathrm{SiO}_{2},{ }^{9,10,26-28}$ with minor changes due to the low spectral energy range of the $\mathrm{Mg} \mathrm{K}$-edge (see the supplementary material). The experiments were performed using the two beams available at the LULI2000 laser facility (École Polytechnique, France). The first beam was employed to drive the shock compression through the sample. Subnanosecond (0.3-0.5 ns) pulses were focused and spatially smoothed with a Hybrid Phase Plate to give a focal spot on a target of $600 \mu \mathrm{m}$ diameter with a flat intensity profile. We varied the energy up to $50 \mathrm{~J}$, for a laser intensity up to $2.9 \times 10^{13}$ $\mathrm{W} / \mathrm{cm}^{2}$. The second laser beam delivered $20 \mathrm{~J}$ at $532 \mathrm{~nm}$ in a $3 \mathrm{ps}$ pulse onto a Gd backlighter, generating a spectrally flat X-ray source around the $\mathrm{Mg} K$-edge $(1.3 \mathrm{keV})$ through $\mathrm{M}$ shell excitation of the $\mathrm{Gd} .{ }^{29,30} \mathrm{Gd}$ was selected as the backlighter material in order to ensure a broad spectrum without any lines, which are often emitted from lower density plasmas. The short duration of the X-ray emission relative to the shock ensures that $\mathrm{MgO}$ is probed in quasistatic conditions. Varying both the drive laser intensity and the delay between the two pulses, allowed us to probe a large range of thermodynamic conditions during $\mathrm{MgO}$ compression and release (see the blue points in Fig. 1).

Our targets consisted of a $0.78 \mu$ m-thick film of boron, a $3.1 \mu \mathrm{m}$-thick polystyrene $(\mathrm{CH})$ ablator, an $\mathrm{MgO}$ sample $(0.5 \mu \mathrm{m}$ $\pm 1 \mathrm{~nm})$, a CVD diamond $(4.1 \mu \mathrm{m} \pm 1 \mathrm{~nm})$, and an additional $\mathrm{CH}$ layer $(3.1 \mu \mathrm{m})$. The target was designed to maximize the transmitted $\mathrm{X}$-ray signal, while allowing us to investigate the $\mathrm{MgO}$ across a wide

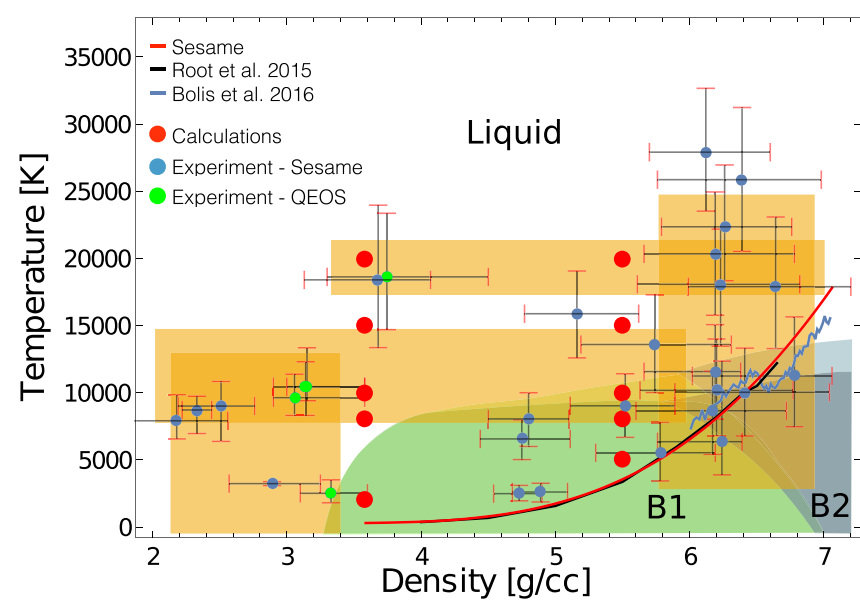

FIG. 1. Thermodynamic conditions probed in MgO with XANES spectroscopy, obtained using MULTI code (for unloading conditions, we show both results obtained with the 7460 SESAME table and QEOS; blue and green dots). The unloading region is affected by an uncertainty due to the choice of EOS. Red dots: conditions obtained with QMD. The orange areas denote the selected isochores and isotherms chosen to investigate the pressure and temperature effects on the electronic and structural properties of $\mathrm{MgO}$. The black and blue lines represent the Hugoniot curves from Refs. 20 and 45, respectively. The red line represents the Hugoniot curve obtained with 7460 SESAME table. B1 and B2 are the two solid phases of $\mathrm{MgO}$. The gray clear area indicates an uncertain region concerning the phase, B2 or liquid following the predictions from Refs. 20 and 21.

range of extreme conditions (see the supplementary material). We characterized the thermal emission from the shock front using Streaked Optical Pyrometry (SOP), with the emission from the rear $\mathrm{CH}$ layer constraining the arrival time of the shock, from which we determined the transit time and thus the mean velocity in the sample layers. This coupled to hydrodynamic simulations performed with the code Multi, ${ }^{31}$ allowed us to determine the thermodynamic states probed by XANES. We performed simulations using two different equations of state: SESAME table and Quotidian Equation of State (QEOS) model. While we did not see significant differences for the compressed states, the unloading conditions are quite sensitive to the choice of the Equation of State (EOS). For this reason, we indicate the two values obtained. Preheating due to X-ray emission in the plasma corona was negligible (see the supplementary material). The XANES spectra were acquired using two independent X-ray spectrometers consisting of truncated-conical RbAP (Rhodamine B and benzyl 3aminipropanoate conjugate), this needs defining crystals and image plates, with a setup similar to those used in Refs. 9, 10, 27, 28, and 32. Raw spectra were corrected for the instrumental response using the procedure described in Refs. 9 and 33.

\section{SIMULATION DESCRIPTION}

Alongside the experimental work, we computed the theoretical XANES spectra using the electronic structure code ABINIT ${ }^{34-36}$ following the methodology described in Refs. 37 and 38. XANES spectra were computed for various thermodynamic conditions, shown by the red dots in Fig. 1, which are similar to those reached in the experiment. The density functional theory was applied with the local density approximation. ${ }^{39}$ The simulations were performed using the projector 
augmented wave (PAW) method. ${ }^{40,41}$ The PAW datasets were generated with the ATOMPAW code ${ }^{42}$ using 10 outer electrons $\left(2 s^{2} 2 p^{6} 3 s^{2}\right)$ and a cutoff radius, $r_{c}=1.9 \mathrm{a}_{B}$, for $\mathrm{Mg}$, and 6 outer electrons $\left(2 s^{2} 2 p^{4}\right)$ and a cutoff radius, $r_{c}=1.2 \mathrm{a}_{B}$, for $\mathrm{O}$. These PAW data sets have previously been benchmarked against the physical properties obtained from the experiments. ${ }^{9,2}$ In order to properly describe the XANES spectra up to $50 \mathrm{eV}$ above the edge, we included high energy projectors. We used a plane wave basis with an energy cut-off of 30 ha. The cubic simulation boxes included 128 atoms for all phases. All molecular dynamics calculations were performed at the $\Gamma$ point for representation of the Brillouin zone, and for XANES calculation, a $3 \times 3 \times 3$ mesh in the Monkorst-Pack scheme ${ }^{43}$ was used. One $\mathrm{Mg}$ atom in the simulation cell was described by a PAW data set including a core hole in the $1 s$ core shell ${ }^{24,44}$ to introduce electron-hole interaction. Each spectrum for a given $(\rho, \mathrm{T})$ condition was an average over 20 configurations. To describe the energy variation of the $1 s$ core orbitals with the density and temperature, we designed an all-electron PAW dataset for Mg. The simulations were performed for 2 atoms in a cubic unit cell with B1 and B2 structures for several densities and temperatures. We then used this computed energy variation of the $1 \mathrm{~s}$ orbitals to recover the shift of the edge for each set of thermodynamical conditions (density/temperature).

\section{RESULTS AND DISCUSSION}

The wide range of probed conditions (see Fig. 1) allowed us to see the individual effects of temperature and density on the XANES spectra by looking at the spectral variation along either isochores and isotherms (orange shaded areas in Fig. 1). Figure 2 shows the measured and calculated XANES spectra along two similar isochores (around $\sim 2.5-3.3 \mathrm{~g} \mathrm{~cm}^{-3}$, AIMD performed at $3.59 \mathrm{~g} \mathrm{~cm}^{-3}$ ) and along the $10000 \mathrm{~K}$ isotherm. Predictions of the spectral evolution along the isochore at high densities $(\sim 6.5 \mathrm{~g} / \mathrm{cc}$ and $5.5 \mathrm{~g} / \mathrm{cc})$ and along the isotherm at $\sim 20000 \mathrm{~K}$ are reported in Figs. S14 and S15 in the supplementary material. Experimental spectra at ambient conditions (labeled "cold" in Fig. 2) are in a good agreement with the theoretical predictions, as well as with the synchrotron data, ${ }^{24,25}$ although the first peak at $1.31 \mathrm{keV}$ is below our experimental resolution (see the supplementary material for a detailed comparison). The ambient $\mathrm{MgO}$ XANES spectrum exhibits strong spectral structures due to the B1 crystalline ordering of $\mathrm{Mg}$ and $\mathrm{O}$ atoms. With increasing temperature, these structures first weaken $(2400 \mathrm{~K})$ and eventually disappear $(9000 \mathrm{~K})$ as the sample melts, but the $\mathrm{Mg} \mathrm{K}$-edge position and shape remain unchanged. By $9000 \mathrm{~K}$, the $\mathrm{MgO}$ XANES spectrum is flat. The calculated spectra exhibit a similar evolution, confirming that the loss a)

$\rho=2.5($ SESAME) $~ 3.3($ QEOS) $\mathrm{g} / \mathrm{cc}$

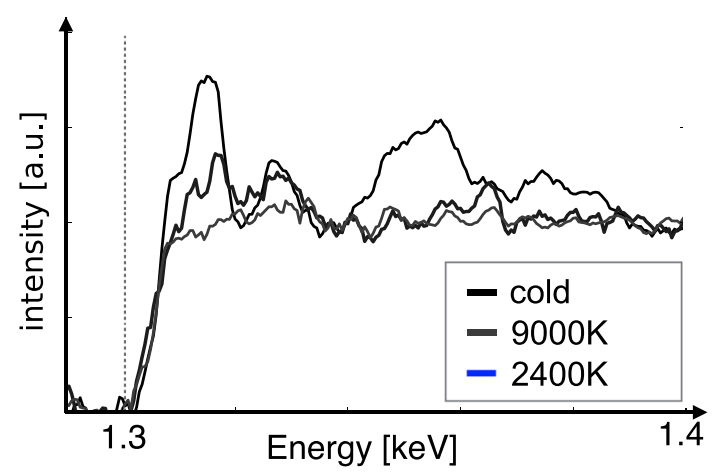

C) $\mathrm{T} \sim 10000 \mathrm{~K}$

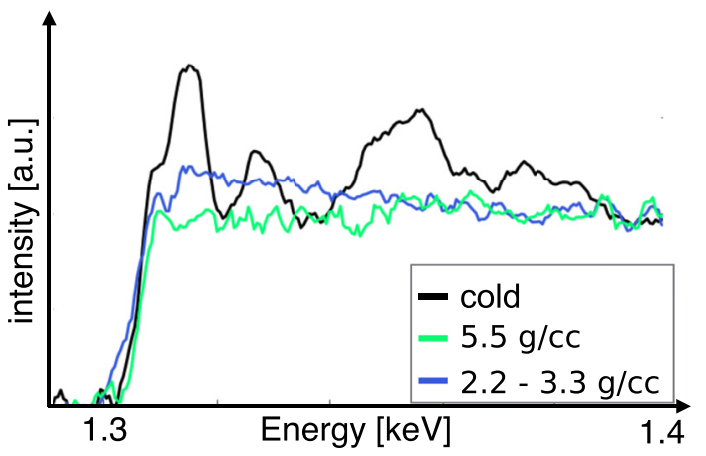

b)

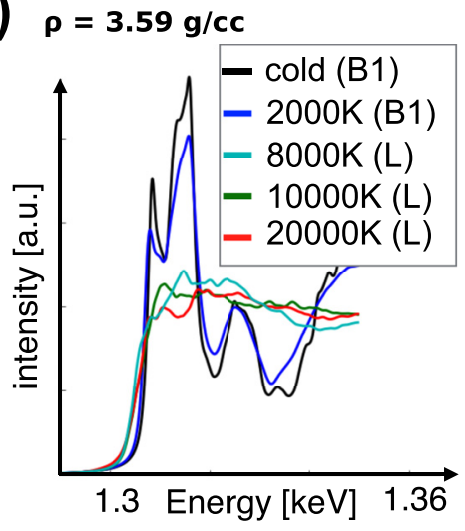

d) $T=10000 \mathrm{~K}$

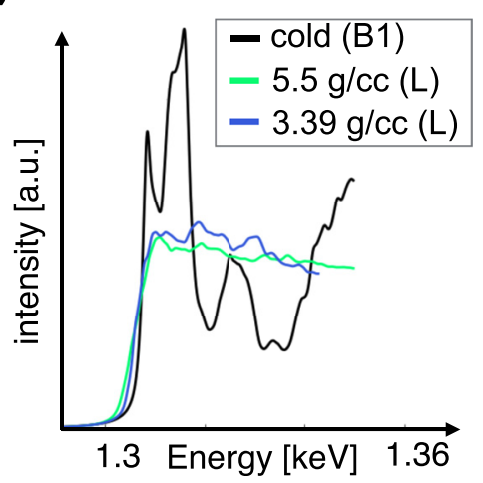

FIG. 2. Measured (left) and calculated (right) XANES spectra along the isochore at a density between $2.5 \mathrm{~g} / \mathrm{cc}$ and $3.3 \mathrm{~g} / \mathrm{cc}$ (obtained with SESAME and QEOS EOS models, respectively) (top) and the isotherm at $\sim 10000 \mathrm{~K}$ (bottom). The significant difference between the calculated spectra in the solid and liquid phases, labeled B1 and L, respectively, is also observed in the measured spectra. 
of spectral structures is due to melting and the associated loss of the structural order. The slight edge broadening in the calculated spectra, which redshifts the edge foot by $\sim 2.4 \mathrm{eV}$, is not observed, since it is below our experimental resolution.

Due to the good agreement between the $a b$ initio predictions and the experimental results, we now examine in more detail the simulations that gave the XANES spectra in Fig. 2(b). Figure 3 shows the density of states (DoS) for temperature points along the isochores at $3.59 \mathrm{~g} / \mathrm{cm}^{-3}$; the solid line gives the total DoS, with the shaded area and dotted line giving the occupied and unoccupied components, respectively. Calculations do not predict a significant variation $(<2.5 \mathrm{eV})$ of the lowest unoccupied state energy between the liquid and solid spectra. As the temperature is not expected to have a significant effect on the energy of core states either, this agrees with a small shift of the $K$-edge which is below our experimental resolution. Although the conduction band broadens slightly with increasing temperature, the foot of the edge moving by $\sim 3 \mathrm{eV}$ is below our experimental resolution $(3.5 \mathrm{eV})$. The data in Fig. 3 also show the process by which the bandgap closes with the temperature. At $2000 \mathrm{~K}$ (i.e., in the solid phase), the electronic bands are broader than those at standard conditions (s.c.), with the bandgap reduced by $\sim 1.5 \mathrm{eV}$, but still open. Above the melting point ( $3125 \mathrm{~K})$, the broadening is large enough so that the bandgap closes and is gradually populated, as shown by the curves corresponding to $5000 \mathrm{~K}$ and $20000 \mathrm{~K}$. Our calculations therefore suggest that $\mathrm{MgO}$ melting and bandgap closure are correlated, in agreement with the previous first principles molecular dynamics results. ${ }^{14}$ The gradual increase in the gap population may correspond to a smooth conductivity increase in liquid $\mathrm{MgO}$, as opposed to materials such as $\mathrm{SiO}_{2}$ where the abrupt increase in the bandgap population causes a discontinuity in conductivity across the melt line.

Turning now to the data along the isotherms at $10000 \mathrm{~K}$ and $20000 \mathrm{~K}$, we can determine the effect of density on the liquid $\mathrm{MgO}$. In these regions of the phase diagram, the changing density has no significant effect on the spectra, which instead remain flat with the $\mathrm{Mg}$

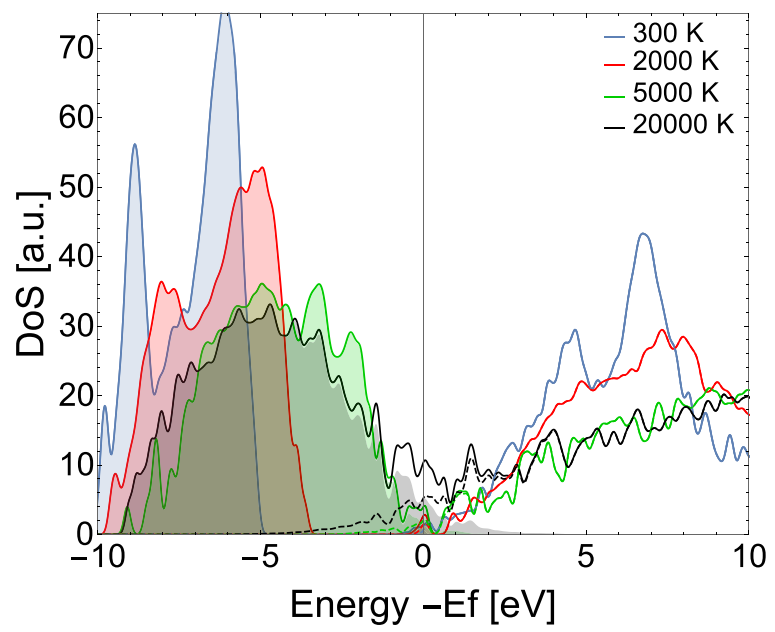

FIG. 3. Calculated electronic density of states along the isochore at $\sim 3.3 \mathrm{~g} \mathrm{~cm}^{-3}$. The continuous lines, filled areas, and dashed lines represent the total, occupied, and unoccupied density of states, at a given temperature, respectively.
$K$-edge remaining unchanged. This is clearly shown in Fig. 2(c), where the density increase from $\sim 2.5-3.3 \mathrm{~g} / \mathrm{cm}^{-3}$ to $5.5 \mathrm{~g} / \mathrm{cm}^{-3}$ does not produce any new spectral features nor induce any shift of the $K$-edge. The AIMD spectra in Fig. 2(d) show the same qualitative behavior, along with a slight $K$-edge broadening which again cannot be resolved.

To understand the reason for this lack of change with the increasing density, we can look at the DoS (Fig. 4) and the AIMD simulations corresponding to the series of XANES spectra along the $10,000 \mathrm{~K}$ isotherm. As the density increases, the DoS in the (closed) bandgap decreases, and the valence band shifts to lower energies. However, the density increase has an almost identical effect on both the core states and the first unoccupied states. Therefore, the difference between the Fermi energy and that of the core states $\mathrm{E}_{F}-\mathrm{E}_{1 s}$ does not change significantly, thus explaining the absence of a $K$-edge shift.

The AIMD calculations also provide an insight into the structural properties of liquid $\mathrm{MgO}$ along the isotherm. The pair correlation functions (PCFs) at $10000 \mathrm{~K}$ and $3.59 \mathrm{~g} / \mathrm{cm}^{-3}$, plotted in Fig. 5 , suggest that molten $\mathrm{MgO}$ is a disordered ionic liquid of $\mathrm{Mg}^{2+}$ and $\mathrm{O}^{2-}$. The like-atom pair correlation functions $g_{\mathrm{MgMg}}(r)$ and $g_{\mathrm{OO}}(r)$ are almost identical, as is typical in an equimolar ionic liquid where anions and cations have the same absolute charge. The first peak of the $\mathrm{Mg}-\mathrm{O}$ pair correlation function $g_{\mathrm{MgO}}(r)$ also partially overlaps with the like-atom PCFs, suggesting that the first neighbor shells contain both unlike and like charged ions. Moreover, angular distributions (see Fig. S11 in the supplementary material) further highlight the absence of a local order in the liquid $\mathrm{Mg}-\mathrm{O}$ distribution, in a good agreement with the observation of flat XANES spectra for temperatures above melting. The disorder is also seen at $5 \mathrm{~g} / \mathrm{cm}^{3}$, with $g_{\mathrm{MgMg}}$ $(r)$ and $g_{\mathrm{OO}}(r)$ being very similar, and $g_{\mathrm{MgO}}(r)$ retaining the same proportions as at a lower density.

The behavior of the $\mathrm{MgO}$ liquid differs strongly from that of $\mathrm{SiO}_{2}$ at similar thermodynamic conditions. Scipioni et al. ${ }^{12}$ showed that, in liquid $\mathrm{SiO}_{2}$ at $3.67 \mathrm{~g} / \mathrm{cm}^{-3}$ and $10000 \mathrm{~K}$, charge ordering organizes atoms at a local level: $\mathrm{Si}$ atoms are enclosed in the first-neighbor shell of $\mathrm{O}$ atoms and vice versa. ${ }^{12}$ This structural character means that the peak in the $\mathrm{SiO}_{2}$ XANES spectra persists to these high temperatures, ${ }^{9,10}$ in notable contrast to our results.

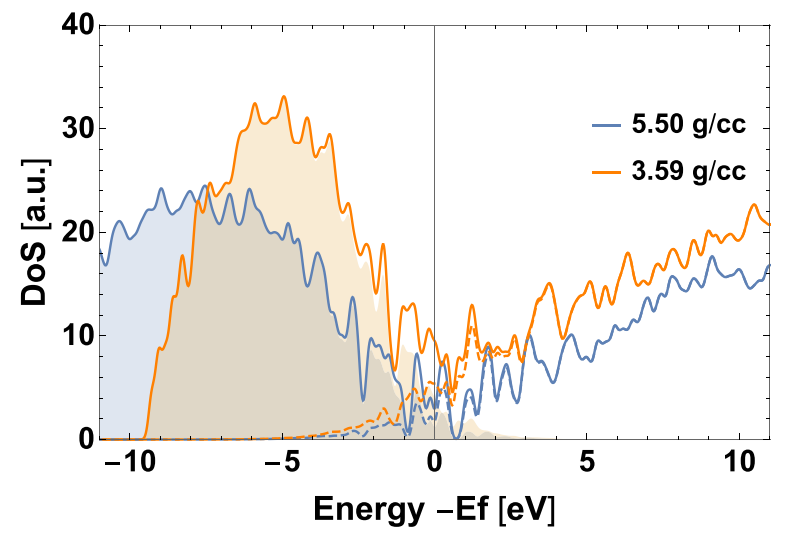

FIG. 4. Calculated electronic density of states along the isotherm at $10000 \mathrm{~K}$. The continuous lines, filled areas, and dashed lines, respectively, represent the total, occupied, and unoccupied density of states. 


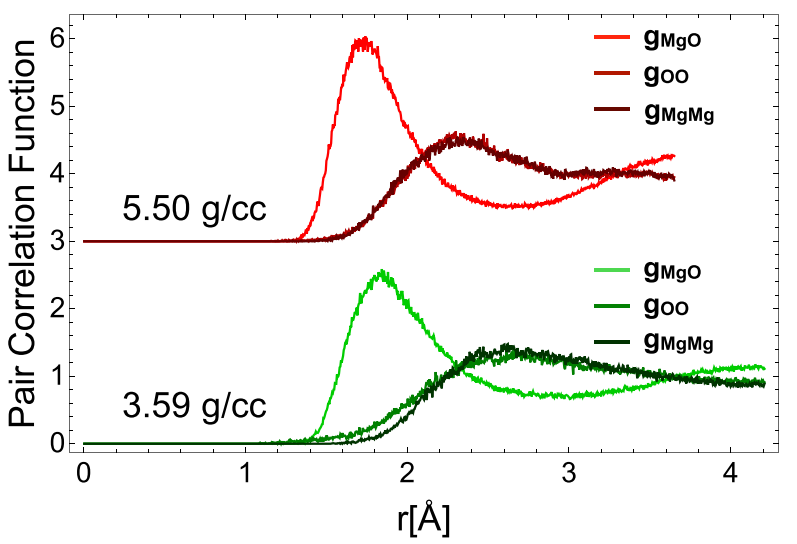

FIG. 5. Computed pair correlation functions at $3.59 \mathrm{~g} \mathrm{~cm}^{-3}$ and $5.5 \mathrm{~g} \mathrm{~cm}^{-3}$, both at $10000 \mathrm{~K}$. An offset has been applied for clarity. At both densities, the material shows a disordered liquid structure.

\section{CONCLUSIONS}

In summary, we have studied the electronic and structural properties of liquid $\mathrm{MgO}$ across a wide range of the warm dense matter phase diagram. Experimental and simulated XANES spectra exhibit the same qualitative behavior with increasing temperature and density, with the calculations providing the means to understand the physical origin of the measured spectral behavior. The analysis of the simulated density of states allowed us to explain that the absence of any shift or strong broadening with increasing temperature was due to the electronic processes due to which the bandgap closes. The temperature has the effect of narrowing the bandgap by creating electronic states energetically located inside the gap, in the vicinity of conduction and valence bands (a phenomenon that appears as a band widening). The DoS also showed that the absence of a shift with increasing density is due to the fact that the difference $\mathrm{E}_{F}-\mathrm{E}_{1 s}$ does not change, even as the values themselves do. This absence of a shift is intuitively consistent with the electronic similarities between defects that populate the bandgap and normal MgO. Structural properties obtained from the analysis of the simulated pair correlation functions are coherent with the absence of structure in the XANES spectra in the liquid phase. At the studied conditions, liquid $\mathrm{MgO}$ demonstrates a strong angular disorder and first neighbor shells which contain both like and unlike charges. The disordered configuration of $\mathrm{Mg}^{2+} \mathrm{O}^{2-}$ ionic pairs prevents photoelectron resonances and thus explains the flat XANES spectra.

\section{SUPPLEMENTARY MATERIAL}

See the supplementary material for more details about the experimental setup and the technical complexities faced to obtain the results described in this paper.

\section{ACKNOWLEDGMENTS}

This research was supported by the ANR Project PLANETLAB (No. ANR-12-BS04-0015) and the ANR Project POMPEI (No. ANR16-CE31-0008). We acknowledge T. Boehly for the helpful and fruitful discussions on data interpretation. We acknowledge the precious support from the technical group at LULI2000.

\section{REFERENCES}

1J. Bouchet, F. Bottin, V. Recoules, F. Remus, G. Morard, R. M. Bolis, and A. Benuzzi-Mounaix, "Ab initio calculations of the B1-B2 phase transition in mgo,” Phys. Rev. B 99, 094113 (2019).

${ }^{2} \mathrm{C}$. Sanchez-Valle and J. Bass, "Elasticity and pressure-induced structural changes in vitreous $\mathrm{MgSiO}_{3}$-enstatite to lower mantle pressures," Earth Planet. Sci. Lett. 295, 523-530 (2010).

${ }^{3}$ D. Pereira, S. Cachinho, M. Ferro, and M. Fernandes, "Surface behaviour of high mgo-containing glasses of the $\mathrm{Si}-\mathrm{Ca}-\mathrm{P}-\mathrm{Mg}$ system in a synthetic physiological fluid," J. Eur. Ceram. Soc. 24, 3693-3701 (2004).

${ }^{4} \mathrm{D}$. Chen and E. Jordan, "Synthesis of porous, high surface area MgO microspheres," Mater. Lett. 63, 783-785 (2009).

${ }^{5}$ T. Duffy, N. Madhusudhan, and K. Lee, Mineralogy of Super-Earth Planets (Elsevier, Oxford, 2015).

${ }^{6}$ L. Ziegler and D. Stegman, "Implications of a long-lived basal magma ocean in generating Earth's ancient magnetic field," Geochem., Geophys., Geosyst. 14, 4735-4742, https://doi.org/10.1002/2013GC005001 (2013).

${ }^{7}$ S. Labrosse, J. Hernlund, and N. Coltice, "A crystallizing dense magma ocean at the base of the earth's mantle," Nature 450, 866 (2007).

${ }^{8}$ L. Stixrude, "Melting in super-earths," Philos. Trans. R. Soc. London A 372, 20130076 (2014).

${ }^{9}$ A. Denoeud, A. Benuzzi-Mounaix, A. Ravasio, F. Dorchies, P. Leguay, J. Gaudin, F. Guyot, E. Brambrink, M. Koenig, S. Le Pape et al., "Metallization of warm dense $\mathrm{SiO}_{2}$ studied by xanes spectroscopy," Phys. Rev. Lett. 113, 116404 (2014).

${ }^{10}$ A. Denoeud, S. Mazevet, F. Guyot, F. Dorchies, J. Gaudin, A. Ravasio, E. Brambrink, and A. Benuzzi-Mounaix, "High-pressure structural changes in liquid silica,” Phys. Rev. E 94, 031201 (2016).

${ }^{11}$ S. Mazevet, T. Tsuchiya, T. Taniuchi, A. Benuzzi-Mounaix, and F. Guyot, "Melting and metallization of silica in the cores of gas giants, ice giants, and super earths," Phys. Rev. B 92, 014105 (2015).

${ }^{12}$ R. Scipioni, L. Stixrude, and M. P. Desjarlais, "Electrical conductivity of $\mathrm{SiO}_{2}$ at extreme conditions and planetary dynamos," Proc. Natl. Acad. Sci. 114, 9009-9013 (2017).

${ }^{13}$ F. Soubiran and B. Militzer, "Electrical conductivity and magnetic dynamos in magma oceans of Super-Earths," Nat. Commun. 9, 3883 (2018).

${ }^{14}$ B. B. Karki, D. Bhattarai, and L. Stixrude, "First-principles calculations of the structural, dynamical, and electronic properties of liquid MgO,” Phys. Rev. B 73, 174208 (2006).

${ }^{15}$ D. Bhattarai, B. B. Karki, and L. Stixrude, "Space-time multiresolution atomistic visualization of $\mathrm{Mgo}$ and $\mathrm{MgSiO}_{3}$ liquid data," Visual Geosci. 11, 1-11 (2006).

${ }^{16}$ B. B. Karki, "First-principles molecular dynamics simulations of silicate melts: Structural and dynamical properties,” Rev. Mineral. Geochem. 71, 355-389 (2010).

${ }^{17}$ N. Van Hong, M. T. Lan, and P. K. Hung, "Structure and dynamics of liquid MgO under high pressure,” High Pressure Res. 32, 509-523 (2012).

${ }^{18} \mathrm{D}$. Cebulla and R. Redmer, " $A b$ initio simulations of $\mathrm{MgO}$ under extreme conditions,” Phys. Rev. B 89, 134107 (2014).

${ }^{19}$ E. Holmström, L. Stixrude, R. Scipioni, and A. Foster, "Electronic conductivity of solid and liquid (Mg, Fe)O computed from first principles," Earth Planet. Sci. Lett. 490, 11-19 (2018).

${ }^{20}$ R. Bolis, G. Morard, T. Vinci, A. Ravasio, E. Bambrink, M. Guarguaglini, M. Koenig, R. Musella, F. Remus, J. Bouchet et al., "Decaying shock studies of phase transitions in $\mathrm{MgO}-\mathrm{SiO}_{2}$ systems: Implications for the super-earths' interiors," Geophys. Res. Lett. 43, 9475-9483, https://doi.org/10.1002/ 2016GL070466 (2016).

${ }^{21}$ R. S. McWilliams, D. K. Spaulding, J. H. Eggert, P. M. Celliers, D. G. Hicks, R. F. Smith, G. W. Collins, and R. Jeanloz, "Phase transformations and metallization of magnesium oxide at high pressure and temperature," Science 338, 1330-1333 (2012).

${ }^{22}$ D. Spaulding, R. McWilliams, R. Jeanloz, J. Eggert, P. Celliers, D. Hicks, G. Collins, and R. Smith, "Evidence for a phase transition in silicate melt at extreme pressure and temperature conditions," Phys. Rev. Lett. 108, 065701 (2012).

${ }^{23}$ O. Fat'yanov, P. Asimow, and T. Ahrens, “Thermodynamically complete equation of state of $\mathrm{MgO}$ from true radiative shock temperature measurements on samples preheated to 1850 K," Phys. Rev. B 97, 024106 (2018). 
${ }^{24}$ R. Nemausat, D. Cabaret, C. Gervais, C. Brouder, N. Trcera, A. Bordage, I. Errea, and F. Mauri, "Phonon effects on X-ray absorption and nuclear magnetic resonance spectroscopies," Phys. Rev. B 92, 144310 (2015).

${ }^{25}$ T. Yoshimura, Y. Tamenori, N. Iwasaki, H. Hasegawa, A. Suzuki, and H. Kawahata, "Magnesium k-edge Xanes spectroscopy of geological standards," J. Synchrotron Radiat. 20, 734-740 (2013).

${ }^{26}$ A. Benuzzi-Mounaix, F. Dorchies, V. Recoules, F. Festa, O. Peyrusse, A. Levy, A. Ravasio, T. Hall, M. Koenig, N. Amadou et al., "Electronic structure investigation of highly compressed aluminum with $\mathrm{k}$ edge absorption spectroscopy," Phys. Rev. Lett. 107, 165006 (2011).

${ }^{27}$ F. Dorchies, F. Festa, V. Recoules, O. Peyrusse, A. Benuzzi-Mounaix, E. Brambrink, A. Levy, A. Ravasio, M. Koenig, T. Hall et al., "X-ray absorption $K$ edge as a diagnostic of the electronic temperature in warm dense aluminum," Phys. Rev. B 92, 085117 (2015).

${ }^{28}$ A. Lévy, F. Dorchies, A. Benuzzi-Mounaix, A. Ravasio, F. Festa, V. Recoules, O. Peyrusse, N. Amadou, E. Brambrink, T. Hall et al., "X-ray diagnosis of the pressure induced mott nonmetal-metal transition," Phys. Rev. Lett. 108, 055002 (2012).

${ }^{29}$ M. Harmand, F. Dorchies, O. Peyrusse, D. Descamps, C. Fourment, S. Hulin, S. Petit, and J. Santos, "Broad $M$-band multi-kev x-ray emission from plasmas created by short laser pulses," Phys. Plasmas 16, 063301 (2009).

${ }^{30} \mathrm{~F}$. Festa, "Etude exp érimentale et théorique de la structure électronique de l'aluminium en conditions extrêmes par spectroscopie d'absorption X," Ph.D. thesis (Ecole Polytechnique X, 2013).

${ }^{31}$ R. Ramis, R. Schmalz, and J. Meyer-ter Vehn, "Multi: A computer code for one-dimensional multigroup radiation hydrodynamics," Comput. Phys. Commun. 49, 475-505 (1988).

${ }^{32}$ A. Levy, F. Dorchies, C. Fourment, M. Harmand, S. Hulin, J. Santos, D. Descamps, S. Petit, and R. Bouillaud, "Double conical crystal x-ray spectrometer for high resolution ultrafast $\mathrm{x}$-ray absorption near-edge spectroscopy of $\mathrm{Al}$ K edge,” Rev. Sci. Instrum. 81, 063107 (2010).

${ }^{33} \mathrm{R}$. M. Bolis, "Investigations of high pressure phase diagrams of $\mathrm{MgO}-\mathrm{SiO}_{2}$ systems with laser shock compression," Ph.D. thesis (Paris Saclay, 2017).

${ }^{34}$ See http://www.abinit.org for The ABINIT code is a common project of the Université Catholique de Louvain, Corning Incorporated, Commissariat à l'Energie Atomique, Université de Liège, Mitsubishi Chemical Corp. and other contributions.
${ }^{35}$ X. Gonze, B. Amadond, P. Anglade, J. Beuken, F. Bottin, P. Boulanger, F. Bruneval, D. Caliste, R. Caracas, M. Côté, T. Deutsch, L. Genovese, P. Ghosez, M. Giantomassi, S. Goedecker, D. Hamann, P. Hermet, F. Jollet, G. Jomard, S. Leroux, M. Mancini, S. Mazevet, M. Oliveira, G. Onida, Y. Pouillon, T. Rangela, G. Rignanese, D. Sangalli, R. Shaltaf, M. Torrent, M. Verstraete, G. Zerah, and J. Zwanziger, "Abinit: First-principles approach to material and nanosystem properties," Comput. Phys. Commun. 180, 2582 (2009).

${ }^{36}$ F. Bottin, S. Leroux, A. Knyazev, and G. Zerah, "Large-scale ab initio calculations based on three levels of parallelization," Comput. Mater. Sci. 42, 329 (2008).

${ }^{37} \mathrm{~V}$. Recoules and S. Mazevet, "Temperature and density dependence of xanes spectra in warm dense aluminum plasmas," Phys. Rev. B 80, 064110 (2009).

${ }^{38}$ F. Dorchies, V. Recoules, J. Bouchet, C. Fourment, P. Leguay, B. Cho, K. Engelhorn, M. Nakatsutsumi, C. Ozkan, T. Tschentscher, M. Harmand, S. Toleikis, M. Störmer, E. Galtier, H. Lee, B. Nagler, P. Heimann, and J. Gaudin, "Time evolution of electron structure in femtosecond heated warm dense molybdenum,” Phys. Rev. B 92, 144201 (2015).

${ }^{39}$ J. P. Perdew and Y. Wang, "Accurate and simple analytic representation of the electron-gas correlation energy,” Phys. Rev. B 45, 13244 (1992).

${ }^{40} \mathrm{P}$. Blochl, "Generalized separable potentials for electronic-structure calculations," Phys. Rev. B 41, 5414 (1990).

${ }^{41} \mathrm{M}$. Torrent, F. Jollet, F. Bottin, G. Zerah, and X. Gonze, "Implementation of the projector augmented-wave method in the ABINIT code: Application to the study of iron under pressure," Comput. Mater. Sci. 42, 337 (2008).

${ }^{42} \mathrm{~N}$. Holzwarth, A. Tackett, and G. Matthews, "A projector augmented wave (PAW) code for electronic structure calculations, Part I: atompaw for generating atom-centered functions," Comput. Phys. Commun. 135, 329 (2001).

${ }^{43}$ H. J. Monkhorst and J. D. Pack, "Special points for Brillouin-zone integrations," Phys. Rev. B 13, 5188-5192 (1976).

${ }^{44}$ V. Mauchamp, M. Jaouen, and P. Schattschneider, "Core-hole effect in the one-particle approximation revisited from density functional theory," Phys. Rev. B 79, 235106 (2009).

${ }^{45}$ S. Root, L. Shulenburger, R. W. Lemke, D. H. Dolan, T. R. Mattsson, and M. P. Desjarlais, "Shock response and phase transitions of $\mathrm{MgO}$ at planetary impact conditions," Phys. Rev. Lett. 115, 198501 (2015). 